\title{
Multi-Feature Based Ocean Oil Spill Detection for Polarimetric SAR Data Using Random Forest and the Self-Similarity Parameter
}

\author{
Shengwu Tong ${ }^{1}$, Xiuguo Liu ${ }^{1}$, Qihao Chen ${ }^{1, * \mathbb{C}}$, Zhengjia Zhang ${ }^{1}$ and Guangqi Xie ${ }^{2}$ \\ 1 Faculty of Information Engineering, China University of Geosciences (Wuhan), Wuhan 430074, China; \\ Cug_tsw@cug.edu.cn (S.T.); liuxiuguo@cug.edu.cn (X.L.); zhangzj@cug.edu.cn (Z.Z.) \\ 2 State Key Laboratory of Information Engineering in Surveying, Mapping and Remote Sensing, \\ Wuhan University, Wuhan 430072, China; xiegqrs@whu.edu.cn \\ * Correspondence: chenqihao@cug.edu.cn; Tel.: +86-27-6788-3728; Fax: +86-27-6788-3809
}

Received: 31 December 2018; Accepted: 18 February 2019; Published: 22 February 2019

\begin{abstract}
Synthetic aperture radar (SAR) is an important means to detect ocean oil spills which cause serious damage to the marine ecosystem. However, the look-alikes, which have a similar behavior to oil slicks in SAR images, will reduce the oil spill detection accuracy. Therefore, a novel oil spill detection method based on multiple features of polarimetric SAR data is proposed to improve the detection accuracy in this paper. In this method, the self-similarity parameter, which is sensitive to the randomness of the scattering target, is introduced to enhance the discrimination ability between oil slicks and look-alikes. The proposed method uses the Random Forest classification combing self-similarity parameter with seven well-known features to improve oil spill detection accuracy. Evaluations and comparisons were conducted with Radarsat-2 and UAVSAR polarimetric SAR datasets, which shows that: (1) the oil spill detection accuracy of the proposed method reaches $92.99 \%$ and $82.25 \%$ in two datasets, respectively, which is higher than three well-known methods. (2) Compared with other seven polarimetric features, self-similarity parameter has the better oil spill detection capability in the scene with lower wind speed close to $2-3 \mathrm{~m} / \mathrm{s}$, while, when the wind speed is close to $9-12 \mathrm{~m} / \mathrm{s}$, it is more suitable for oil spill detection in the downwind scene where the microwave incident direction is similar to the sea surface wind direction and performs well in the scene with incidence angle range from $29.7^{\circ}$ to $43.5^{\circ}$.
\end{abstract}

Keywords: oil spill detection; polarimetric SAR; self-similarity parameter; random forest; multi-feature

\section{Introduction}

\subsection{Background}

With the development of the marine transport and offshore oil industries, oil spills caused by leaks in pipelines, offshore oil tankers, and drilling platforms occur more often. Large amounts of crude oil and refined oil enter the ocean in different ways, causing significant damage to birds, marine organisms and coastal environments. Hence, it is very necessary to monitor marine oil spills, which is of great help for pollution treatment. Remote sensing plays an increasingly important role in oil spill response efforts, as the location and extent of oil spills can be quickly and accurately determined by remote sensing [1]. Among many remote sensing sensors, synthetic aperture radar (SAR) is an effective tool of oil spill detection due to its all-weather and all-day capabilities [2-4].

On the ocean's surface, capillary waves and short gravity waves of water reflect microwave energy, producing a "bright" image known as sea clutter in single-polarization SAR image [1]. The oil slicks on the ocean surface dampen capillary waves and short gravity waves, which cause microwave 
backscatter energy of oil slicks weaker than the surrounding clean sea. Therefore, oil slicks are normally detected as dark areas in the SAR images [5-7]. However, there are other natural phenomena which cause dark areas in SAR images, like natural biogenic slicks, rain cells, internal waves, grease ices, currents, etc. $[6,8,9]$. These look-alike phenomena increase the difficulty of oil spill detection. Compared with single-polarization SAR, polarimetric SAR includes not only the power information but also the relative phase information between channels, which can reflect the difference in target characteristics. Many studies have proven that the extra polarimetric information contained in polarimetric SAR data can provide powerful discrimination between oil slicks and look-alikes [3,10-12].

\subsection{Related Works}

There are many studies of oil spill detection using polarimetric features from polarimetric SAR data in recent years, which detect oil slicks by the differences of polarimetric information between oil slicks and look-alikes. Many polarimetric features have been proposed to detect oil spills [13]. These features are mainly divided into three categories, according to their principle of oil spill detection.

The first kind utilizes the backscattered energy to distinguish oil slicks; for example, span [14], Freeman decomposition parameters [15], geometric intensity $(V)$ [16], Bragg energy proportion $(\eta)$ [17], etc. Among them, $V$ is classified in this category because it is mathematically similar to the span, but it contains information on the cross products in addition to the intensities used in the span [16]. $\eta$ refers to the ratio of Bragg scattering energy to the total energy in the microwave reflected energy [17].

The second kind utilizes the correlation between different channels to distinguish oil slicks; for example, the standard deviation of the co-polarized phase difference ( $c p d)$ [4], the real part of the co-polarization cross product (Rco) [16], conformity coefficient ( $\mu$ ) [18], etc. Among them, Rco was proposed that has the ability to distinguish slick-covered areas from the clean sea and look-alikes [16,19].

The third kind utilizes the scattering mechanism to distinguish oil slicks-for example, $H / \alpha / A$ decomposition parameters [7], modified anisotropy coefficient $\left(A_{12}\right)$ [16], etc. In addition, some features are also classified into this category that can detect oil spills by measuring polarized or unpolarized components in the electromagnetic waves, such as the degree of polarization $(D o P)$ [20], pedestal height $(P h)[3]$, etc. Among them, Dop can measure how close the scattering mechanism of the observed scene is to be deterministic [20], and $P h$ is a reliable estimator of the amount of unpolarized backscattered energy [3].

In addition to the polarimetric SAR, compact-polarimetric SAR has also been introduced for oil spill detection [21-23]. Some above-mentioned full-polarimetric features were extended to compact-polarimetric SAR for oil spill detection, such as $\mu[10,24], \operatorname{DoP}[10,25]$, etc. In this paper, we focus on the fully polarimetric features.

Among these features introduced above, some show better ability of oil spill detection, which has already been proved in related literature $[3,16,18,20]$. The expressions for these features, along with their expected behavior over the oil slicks and look-alikes, are described in Table 1.

With the increasing number of polarimetric features utilized for oil spill detection, it gradually becomes a trend that uses multiple features to detect oil spills [13]. For example, Liu et al. proposed a parameter $\mathrm{F}$, which is a combination of multi-feature, combined with Otsu segmentation to detect oil spills [14]. Yin et al. proposed an extended Bragg model combined with maximum likelihood classification for oil spill detection [26]. Ramsey et al. used Freeman-Durden decomposition and Cloude-Pottier decomposition combined with Wishart classification for oil spill detection [15], etc. In consideration of the complementarity among features to the classification performance, it may be possible to use multi-features simultaneously to improve the oil spill detection accuracy and reduce the false alarm caused by look-alikes [11,27]. 
Table 1. Polarimetric features and their expected behavior over the oil slicks and look-alikes.

\begin{tabular}{cccc}
\hline Categories & Polarimetric Features & Oil Slicks & Look-Alikes \\
\hline Backscattered energy & $\eta=\frac{T_{11}+\left|T_{12}\right|^{2} / T_{11}}{S p a n}$ & Low & High \\
$V=\operatorname{det}(\boldsymbol{T})^{3}$ & Low & High \\
\hline $\begin{array}{c}\text { Correlation between } \\
\text { different channels }\end{array}$ & $\begin{array}{c}\mu=\frac{2\left(\operatorname{Re}\left\langle S_{H H} S_{V V}^{*}\right\rangle-\left|S_{H V}\right|^{2}\right)}{S p a n} \\
\text { Rco }=\left|\operatorname{Re}\left(\left\langle S_{H H} S_{V V}^{*}\right\rangle\right)\right|\end{array}$ & Low & High \\
$\begin{array}{c}\text { Scattering mechanism or } \\
\text { polarized state }\end{array}$ & $D o P=\sqrt{\frac{1}{3}\left(\frac{\operatorname{tr}\left(\boldsymbol{M}^{t} \boldsymbol{M}\right)}{M_{11}^{2}}-1\right)}$ & Low & High \\
\hline $\begin{array}{c}P h=\frac{\lambda_{3}}{\lambda_{1}} \\
A_{12}=\frac{\lambda_{1}-\lambda_{2}}{\lambda_{1}+\lambda_{2}}\end{array}$ & High & High \\
\end{tabular}

where $T_{i j}$ means the $(i, j)$ entry of coherency matrix $T, \operatorname{Span}$ denotes the total power of scattering matrix, $\operatorname{det}(\cdot)$ is the determinant, $\operatorname{Re}(\cdot)$ denotes the real part, $S_{H H}, S_{H V}$, and $S_{V V}$ are the complex elements of the scattering matrix, * means the complex conjugate operator, $\langle\cdot\rangle$ is the ensemble average operator, $\operatorname{tr}(\cdot)$ is the trace operator of the matrix, $\boldsymbol{M}$ means the Mueller matrix, and $M_{i j}$ is the $(i, j)$ entry of $\boldsymbol{M}, t$ stands for transpose operator, $\lambda_{i}$ is eigenvalue of $\boldsymbol{T}$.

\subsection{The Proposed Approach}

The Random Forest (RF) algorithm based on multi-feature has proven to have a good performance, which is robust to noise and outliers without over-fitting [28,29]. In addition, the RF can also assess the importance of the feature [30,31]. In this paper, we propose a multi-feature based method using RF classification to detect oil spills from polarimetric SAR data. Three kinds of polarimetric features shown in Table 1 were used for oil slick classification.

In order to improve the oil spill detection accuracy and reduce the errors caused by look-alikes, we also introduce a new polarimetric feature, self-similarity parameter, to enhance the discrimination ability between oil slicks and look-alikes. The self-similarity parameter can be used to describe the randomness of the scattering mechanism of the target [32]. Compared to water and look-alikes, the randomness of scattering mechanisms in oil slicks is higher because of its complex scattering mechanism [33]. Hence, the self-similarity parameter was introduced to detect oil spills along with other seven polarimetric features.

This paper is organized as follows: Section 2 introduces the method frame of oil spill detection. The experimental data, oil spill detection ability analysis of different polarimetric features, and comparison of different methods are introduced in Section 3. The noise analysis and influencing factors of oil spill detection are analyzed in Section 4. Section 5 introduces the major conclusions of this paper.

\section{Methodology}

\subsection{Method Frame for Oil Spill Detection}

The flow chart of the proposed method is shown in Figure 1. It mainly includes two parts, the first part is the polarimetric features extraction, and the second part is multi-feature based RF classification. In order to suppress the effect of speckle noise, the polarimetric SAR images were processed by Lee filtering with a $7 \times 7$ window size.

In the first part, the self-similarity parameter and seven other polarimetric features shown in Table 1 were extracted. After features extraction, multi-feature fusion was performed as the input variable for RF.

In the second part, the RF classification was used to classify oil slicks. First, obtain the multiple bootstrap datasets by multiple random sampling from the training sample (i.e., oil slicks, look-alikes, and water). Then, construct the decision tree for each bootstrap dataset, grow the tree using Classification and Regression Trees (CART) to maximum size and do not prune [34]. Then, use multiple decision trees to build the random forest model of oil spill detection and use voting classification 
to classify oil slicks, look-alikes, and water in the images. Finally, detect oil spills according to the classification results.

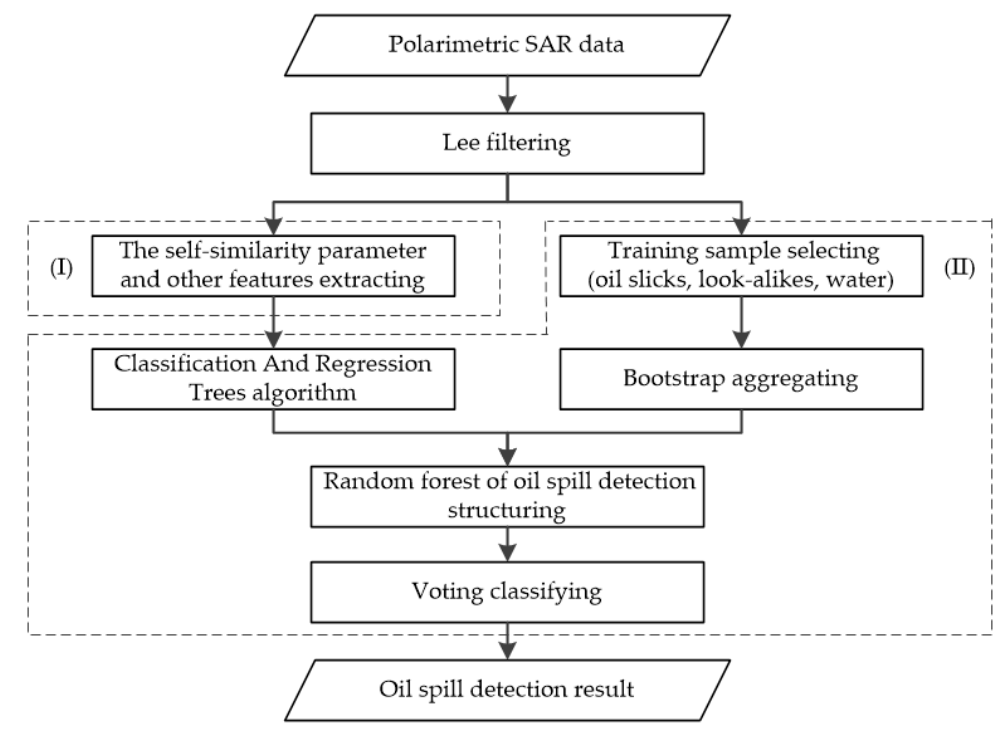

Figure 1. The flow chart of the proposed method.

\subsection{Using Self-Similarity Parameters to Describe Oil Slicks}

In Section 2.1, we introduced the processes of the proposed method. Among them, the polarimetric features were as the inputs of the RF classification. In-depth explanations of seven features in Table 1 have been provided in the corresponding literature $[3,16,18,20]$. Thus, this section focuses on explaining the self-similarity parameter.

The similarity parameter was proposed to measure the similarity between two scattering matrices by Yang et al. [35], which extracts the scattering mechanism of targets by calculating the similarity between targets and the canonical target (i.e., sphere, diplane reflector, left/right helix, etc.) $[35,36]$. The self-similarity parameter $\left(r r r_{s}\right)$ is a new parameter defined based on the similarity parameter by Li et al., which is related to its own scattering matrix and not associated with the canonical target [32]. It is used to describe the randomness of the target scattering mechanism.

The full polarimetric SAR system measures four combinations of liner transmit and receive polarization, the scattering matrix $S$ of is:

$$
S=\left[\begin{array}{ll}
S_{H H} & S_{H V} \\
S_{V H} & S_{V V}
\end{array}\right] .
$$

The matrix $S$ is a symmetric matrix under the monostatic radar system, i.e., $S_{H V}=S_{V H}$. The Pauli scattering vector $k$ can be extracted from the scattering matrix $S$ as:

$$
\boldsymbol{k}=\frac{1}{\sqrt{2}}\left[S_{H H}+S_{V V}, S_{H H}-S_{V V}, 2 S_{H V}\right]^{t} .
$$

As for two given targets, their similarity parameter $r$ based on matrix $S$ is defined as [35]:

$$
r\left(S_{1}, S_{2}\right)=\frac{\left|\boldsymbol{k}_{1}{ }^{H} \boldsymbol{k}_{2}\right|^{2}}{\left\|\boldsymbol{k}_{1}\right\|^{2}\left\|\boldsymbol{k}_{2}\right\|^{2}},
$$

where $H$ is conjugate transpose operator, and $\|\cdot\|^{2}$ denotes the square sum of the absolute values of the components of the vector. 
In practical applications, the polarimetric coherence matrix $T$ is generally used to describe the distributed target. In this case, the similarity parameter $r r r$ of a mixed scatterer $T$ and a canonical mixed scatterer $\boldsymbol{T}_{\mathcal{C}}$ based on coherence matrix $\boldsymbol{T}$ can be described as [32]:

$$
\operatorname{rrr}\left(\boldsymbol{T}, \boldsymbol{T}_{c}\right)=\frac{\operatorname{tr}\left(\boldsymbol{T} \boldsymbol{T}_{c}^{H}\right)}{\operatorname{tr}(\boldsymbol{T}) \operatorname{tr}\left(\boldsymbol{T}_{\mathcal{c}}\right)}=\frac{\sum_{i=1}^{3} \lambda_{i} \lambda_{c i}}{\sum_{i=1}^{3} \lambda_{i} \sum_{i=1}^{3} \lambda_{c i}}
$$

In order to measure the randomness of target scattering, the self-similarity parameter is obtained from similarity parameter $r r r$ when $\boldsymbol{T}=c \boldsymbol{T}_{c}$, where $c$ is an arbitrary number. Therefore, the self-similarity parameter $r r r_{s}$ of matrix $T$ is defined as [32]:

$$
\operatorname{rrr}_{\mathcal{S}}(\boldsymbol{T})=\operatorname{rrr}\left(\boldsymbol{T}, \boldsymbol{T}_{\mathcal{c}}\right)=\frac{\sum_{i=1}^{3} \lambda_{i} \lambda_{c i}}{\sum_{i=1}^{3} \lambda_{i} \sum_{i=1}^{3} \lambda_{c i}}=\frac{\sum_{i=1}^{3} \lambda_{i}^{2}}{\left(\sum_{i=1}^{3} \lambda_{i}\right)^{2}}=\frac{\operatorname{tr}\left(\boldsymbol{T} \boldsymbol{T}^{H}\right)}{(\operatorname{tr}(\boldsymbol{T}))^{2}}
$$

The value of the $r r r_{s}$ ranges from 1/3 to 1 . When the scattering mechanism of the target is more single, the value of $r r r_{S}$ is larger, and the value of $r r r_{S}$ reaches the maximum 1 when the scattering mechanism is the single scatterer, while, when the scattering mechanism of the target is more complex, the value of $r r r_{s}$ is smaller, and the value of $r r r_{s}$ reaches the minimum 1/3 when the scattering mechanism is the randomly noisy scatterer.

The value distribution of three eigenvalues in the oil slicks, look-alikes, and water are shown in Figure 2a. The scattering mechanism of the slick-free sea surface is mainly the Bragg or titled Bragg scattering [3], its first eigenvalue $\lambda_{1}$ is much larger than the second eigenvalue $\lambda_{2}$ and the third eigenvalue $\lambda_{3}$ as shown in Figure 2a. Hence, the value of $r r r_{s}$ in the water region is relatively high. In the oil slicks, the $\lambda_{1}$ is close to the $\lambda_{2}$ and $\lambda_{3}$, the value of $r r r_{s}$ in the oil slick region is relatively low, for the scattering mechanism is complex scattering with high depolarization, due to the strong damping properties of the oil slicks [3]. In the look-alikes, taking natural biogenic slicks as an example, its scattering mechanism is still Bragg scattering, although characterized by low backscatter returns, which generate a dark area in the SAR image $[3,37,38]$. Thus, the $\lambda_{1}$ of look-alikes is still much larger than the $\lambda_{2}$ and the $\lambda_{3}$, the value of $r r r_{s}$ of the look-alike region is still high compared to the oil slick area.

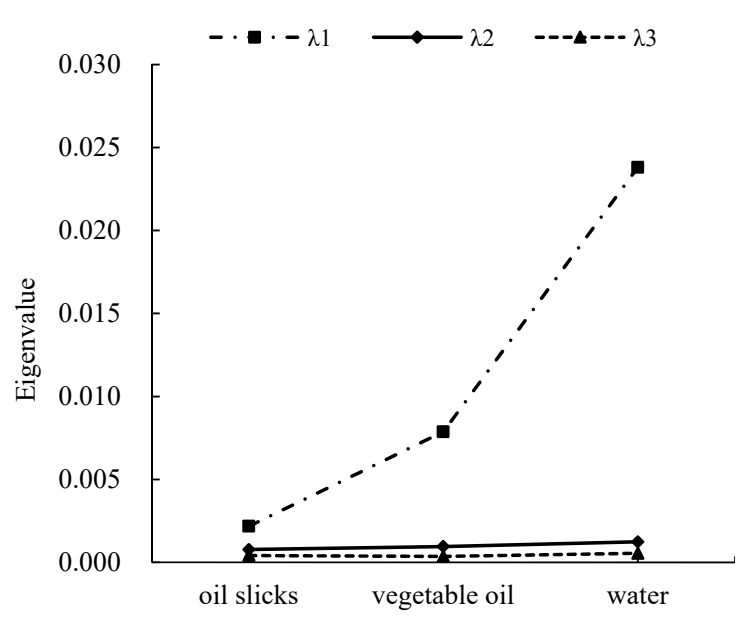

(a)

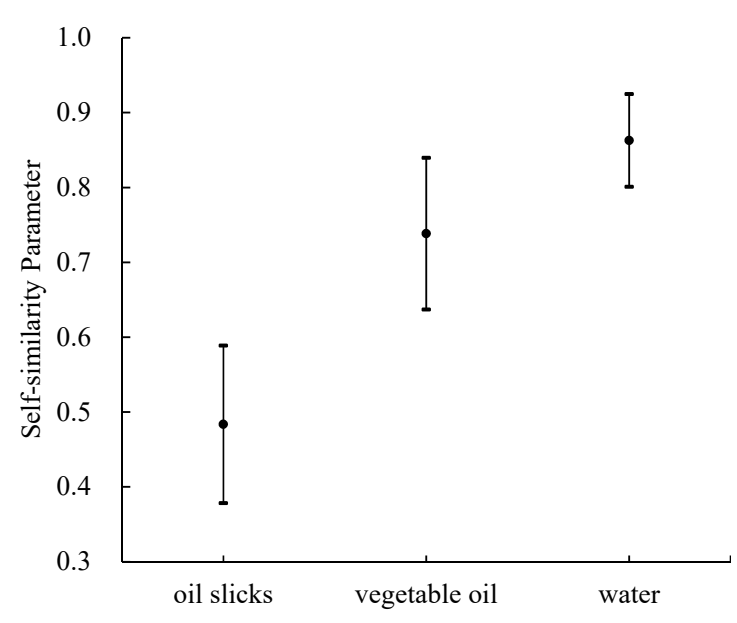

(b)

Figure 2. The value of features in the oil slicks, look-alikes, and water. (a) three eigenvalues; (b) self-similarity parameter.

In order to visually represent $r r r_{s}{ }^{\prime}$ difference among oil slicks, look-alikes, and water, the mean and standard deviations of $r r r_{s}$ in different targets are shown in Figure $2 b$, according to the samples 
shown in Figure 3. The vertical bars show the mean and standard deviation of the value of the $r r r_{S}$ in four regions. It is obvious that there is a good distinction between oil slicks and look-alikes. Since $r r r_{S}$ detect oil slicks based on the randomness of the scattering mechanism, it should be classified as the third category in Table 1, namely "Scattering mechanism or polarized state".

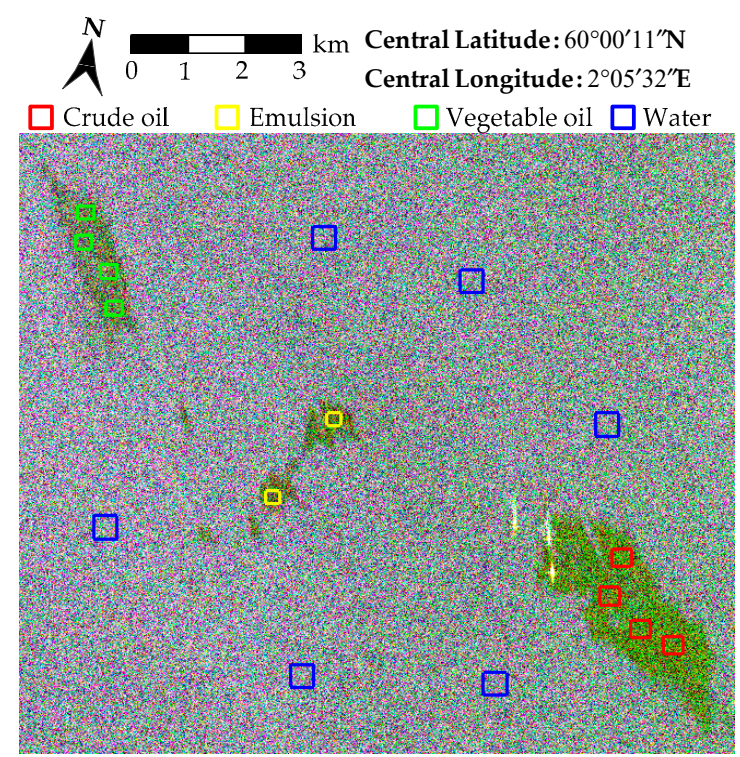

Figure 3. Pauli color-coded image of the Radarsat-2 polarimetric SAR image as the first dataset.

\subsection{Oil Spill Detection Based on RF Classification}

Based on the above eight polarimetric features as the inputs, RF is utilized to classify oil slicks in the proposed method. It is an integrated learning algorithm proposed by Breiman [34], which has been used in polarimetric SAR image classification $[29,39,40]$. RF classification consists of multiple decision trees, which generated based on a combination of a random subsample of the training dataset and a random subset of explanatory input features.

In the ocean oil spill detection, water usually occupied most of the image, which caused class imbalance and interfered with the final classification accuracy. Under this circumstance, RF can suppress the classification error effectively [34]. In addition, RF can effectively suppress over-fitting caused by noise and erroneous samples [41,42], and RF can process large amounts of data without features selection. Additionally, RF provides a variable importance (VI) index to estimate the contribution of each feature, which is of good help for analyzing which features are better for oil spill detection [29].

The eight polarimetric features described above are used as the inputs of RF classification, and the training data used in the RF model learning process are also selected among these features.

The oil spill detection steps based on RF classification are as follows:

(1) Randomly select partial subsets from total training data to obtain the multiple bootstrap datasets, which include oil slicks, look-alikes, and water. Use these bootstrap datasets to construct decision trees.

(2) Randomly select partial features from inputs at each node of each decision tree, and then select one of these features which has the best classification ability to determine the split.

(3) Each tree grows to the maximum using CART, without any cutting in the process of division.

(4) Use the RF model that combines multiple decision trees to vote classification with the input polarimetric features. After the classification, the look-alikes and water are masked, and the oil slicks are the final result of oil spill detection.

In this study, the RF classification implemented in "imageRF" and available as part of the EnMAP-Box distribution was used. 


\section{Experiment and Results}

\subsection{Experimental Data}

In this paper, two datasets, Radarsat-2 and Uninhabited Aerial Vehicle Synthetic Aperture Radar (UAVSAR), were used to verify the proposed method. The details of these experiment datasets are shown in Table 2.

Table 2. The details of the two experiment datasets.

\begin{tabular}{cccccc}
\hline Sensor & Number & Date, Time UTC & Relative Direction & Wind Speed & Incidence Angle \\
\hline Radarsat-2 & $/$ & 8 June 2011 17:27 & $/$ & $1.6-3.3 \mathrm{~m} / \mathrm{s}$ & $35.1-35.9^{\circ}$ \\
\hline \multirow{3}{*}{ UAVSAR } & $\# 1$ & 10 June 2015 06:13 & Downwind & $9-11 \mathrm{~m} / \mathrm{s}$ & $39.6-43.5^{\circ}$ \\
& $\# 2$ & 10 June 2015 07:05 & Downwind & $9-11 \mathrm{~m} / \mathrm{s}$ & $29.7-34.4^{\circ}$ \\
& $\# 3$ & 10 June 2015 07:17 & Upwind & $9-11 \mathrm{~m} / \mathrm{s}$ & $28.9-34.2^{\circ}$ \\
\hline
\end{tabular}

Notes: The "Relative direction" refers to the look direction relative to the wind. In the case of UAVSAR data acquisition, the aerial vehicle has two headings, ascending pass and descending pass. Since the sensor is left-looking and the measured average wind direction is almost constant during the experiment, the sensor was looking close to upwind for the ascending pass and closed to downwind for the descending pass. The "Incidence angle" refers to the range of incidence angle in the oil slick and look-alike region.

The first dataset is a C-band Radarsat-2 image with a resolution of $5.2 \mathrm{~m} \times 7.6 \mathrm{~m}$, which was collected from a man-made experiment of oil spills by Norwegian Clean Seas Association for Operating Companies (NOFO). Three different kinds of film were released onto the surface, vegetable oil, emulsion, and crude oil. Among them, approximately $0.4 \mathrm{~m}^{3}$ Radiagreen ebo vegetable oil was used to simulate the natural monomolecular biogenic slick, since the vegetable oil has a similar structure as the surface-active compounds in natural biogenic slicks. There is approximately $1 \mathrm{~m}^{3}$ of emulsion on the surface, which consists of Oseberg blended crude oil and IFO380. The crude oil consists of $30 \mathrm{~m}^{3}$ of evaporated Balder crude oil. These slicks were sprinkled onto the sea surface $13 \mathrm{~h}, 29 \mathrm{~h}$, and $9 \mathrm{~h}$ before satellite imaging, respectively [16]. The Pauli color-coded image of Radarsat-2 is shown in Figure 3, the red box is the crude oil region, the yellow box is the emulsion region, the green box is the vegetable oil region, and the blue box is the water region.

The second dataset contains three L-band UAVSAR images (https:/ / uavsar.jpl.nasa.gov/), with a resolution of $7.2 \mathrm{~m} \times 5 \mathrm{~m}$. They were collected during the Norwegian Radar Oil Spill Experiment (NORSE 2015). There are four kinds of film in the image, including three different emulsions and one vegetable oil, were released onto the sea surface close in time. Among them, the emulsions were all based on Troll and Oseberg crude oils but had different oil volumetric fractions, i.e., 80\% oil (E80), $60 \%$ oil (E60), and 40\% oil (E40) [43]. The vegetable oil was the Radiagreen ebo, which is used to simulate the biogenic slicks. The volumes of the releases were $0.5 \mathrm{~m}^{3}$ for each of the emulsions and $0.2 \mathrm{~m}^{3}$ for the vegetable oil. The Pauli color-coded image of \#1 in the dataset is shown in Figure 4.

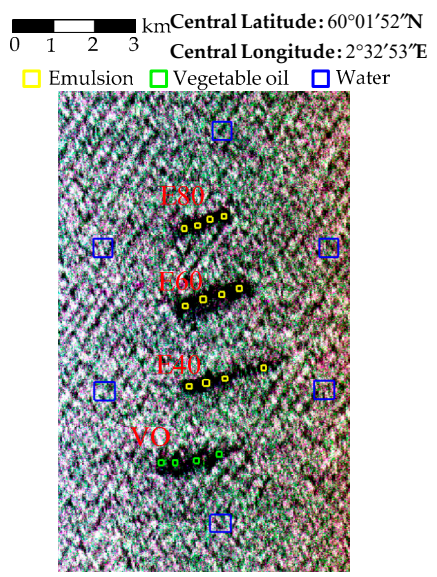

Figure 4. Pauli color-coded image of the UAVSAR polarimetric SAR image in the second dataset. 


\subsection{Oil Spill Detection Ability Analysis of the Self-Similarity Parameter}

In order to verify the ability of a self-similarity parameter for oil spill detection, we compared the self-similarity parameter and other seven polarimetric features from both the qualitative analysis (probability density function of these features, the results of oil slicks classification using these features separately) and quantitative analysis (Jeffreys-Matusita distance of these features) aspects using the Radarsat-2 image.

The probability density function (pdf) of the eight polarimetric features in four areas: crude oil (c), emulsion (e), vegetable oil (v), and water (w), are shown in Figure 5, the samples are shown in Figure 3. The features are here normalized for the whole image. In the pdfs, more overlap between the two regions means the weaker discriminability of the two regions; less overlap means the stronger discriminability. Overall, in the eight features, there are few overlap regions between the oil slicks and the water, which means that the oil slicks and the water have a good distinction in these polarimetric features. The overlap regions between two oil slicks are relatively large, and a separation between these two is hard based on these features. There are few overlap regions between the oil slicks and the vegetable oil. Among them, the overlap areas between the crude oil and the vegetable oil are less than that between the emulsion and vegetable oil except for Figure 5f. In addition, it is obvious that the overlap regions between the crude oil and the vegetable oil in Figure $5 e, g$, and $h$ are much few than that in Figure $5 a, b, d$, and that in Figure $5 c, f$ are similar. The overlap regions between the emulsion and the vegetable oil in Figure 5e-h are few than that in Figure 5a-d.

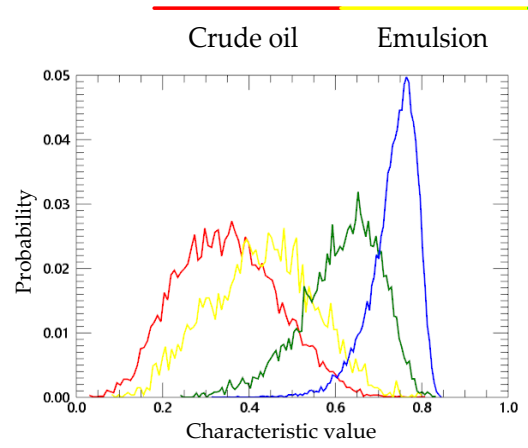

(a)

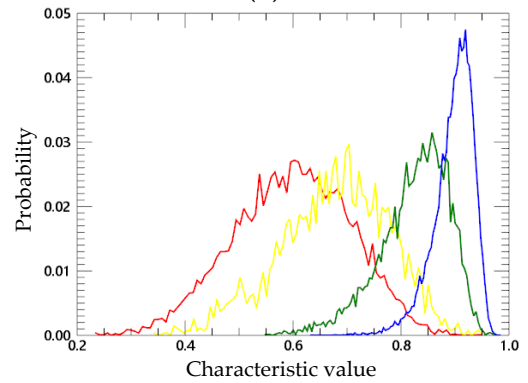

(b)

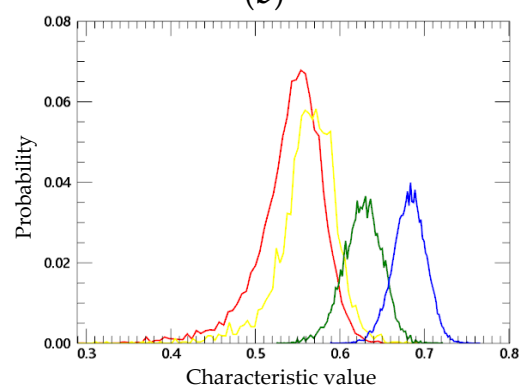

(c)

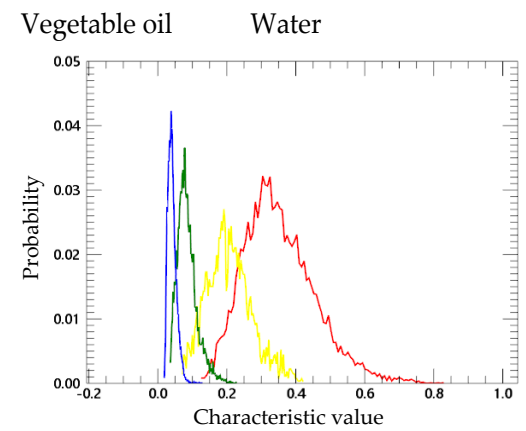

(e)

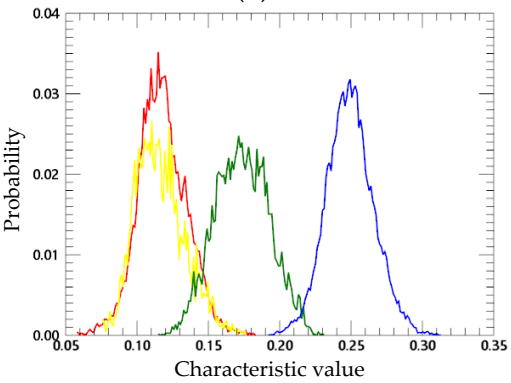

(f)

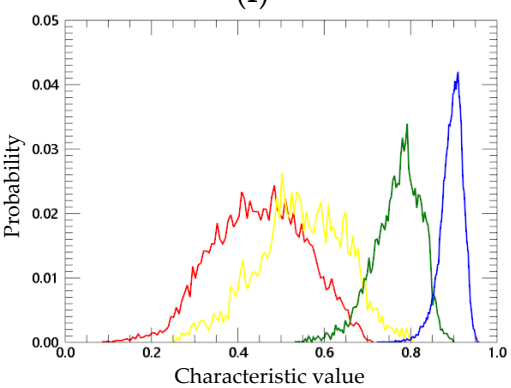

(g)

Figure 5. Cont. 


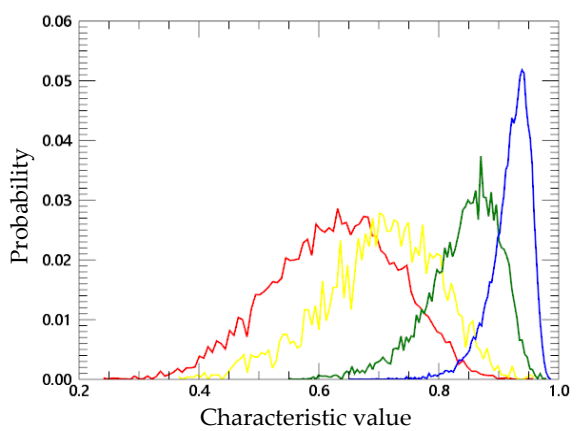

(d)

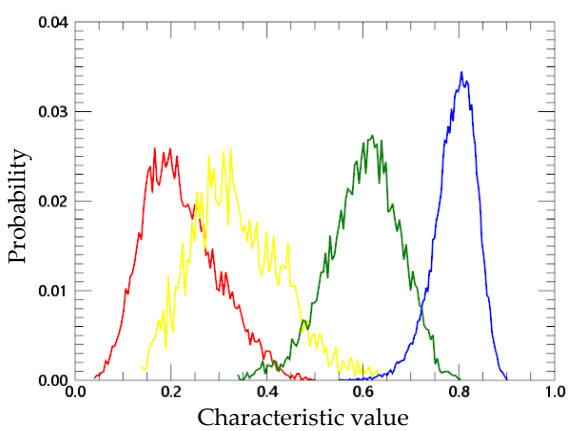

(h)

Figure 5. The Probability Density Function (PDF) of eight polarimetric features values within selected regions of Radarsat-2 images. The features $V$ and $R c o$ are $\log$ transformed for visualization purposes; (a) $\mathrm{DoP} ;$ (b) $\mu$; (c) $\log R c o ;(\mathbf{d}) \eta$; (e) $P h ;$ (f) $\log V$; (g) $A_{12}$; and (h) $r r r_{s}$.

In order to visually represent the effect of each feature on the oil spill detection, the classic $k$-means classifications based on these individual features are compared. The unsupervised classification results are shown in Figure 6. In all cases, the main parts of the emulsion and crude oil are classified as the red class, and the surrounding zones of them are classified to the green class. The main parts of the water are classified to the blue class and mixed with some green class. In addition, vegetable oil in the upper left corner is mainly classified to the green class and mixed with the different degrees of the red class and blue class. It is obvious that the number of red classes in the vegetable oil area in Figure $6 \mathrm{e}-\mathrm{h}$ is less than that in Figure 6a-d.

\section{Oil slick Look-alike Water}

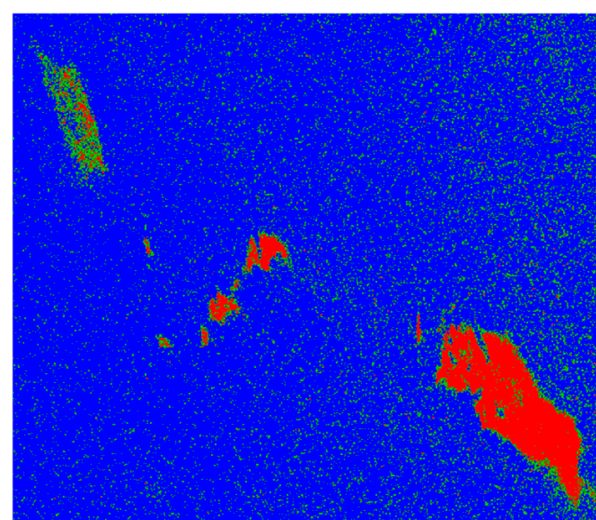

(a)

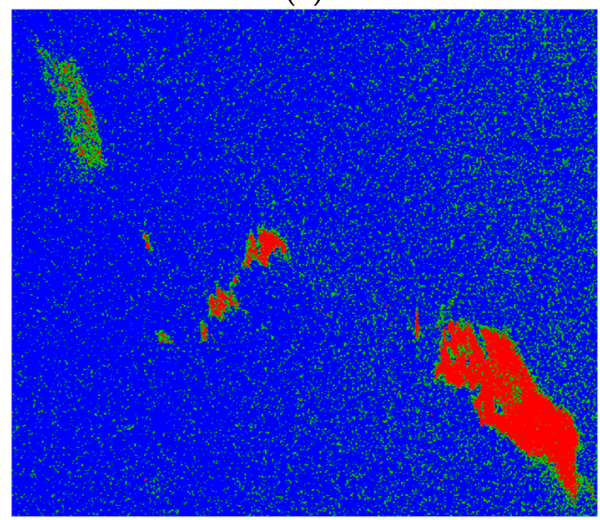

(b)

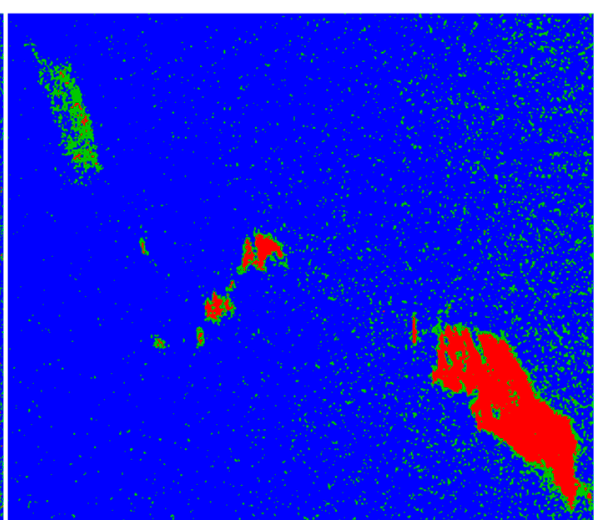

(e)

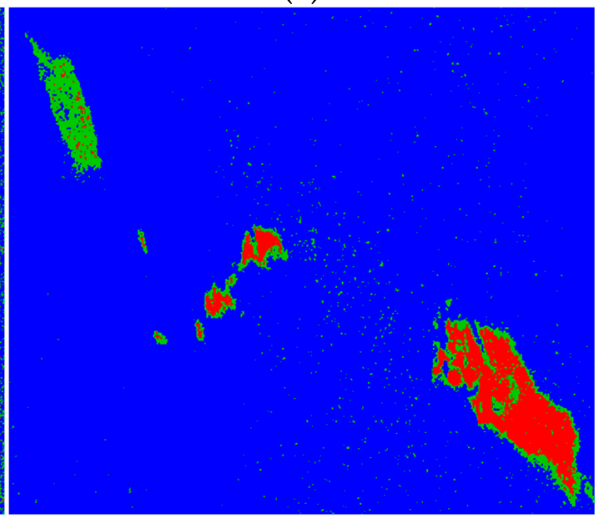

(f)

Figure 6. Cont. 


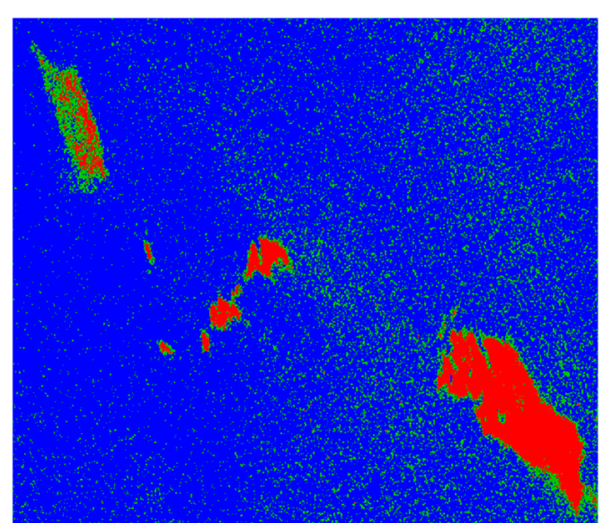

(c)

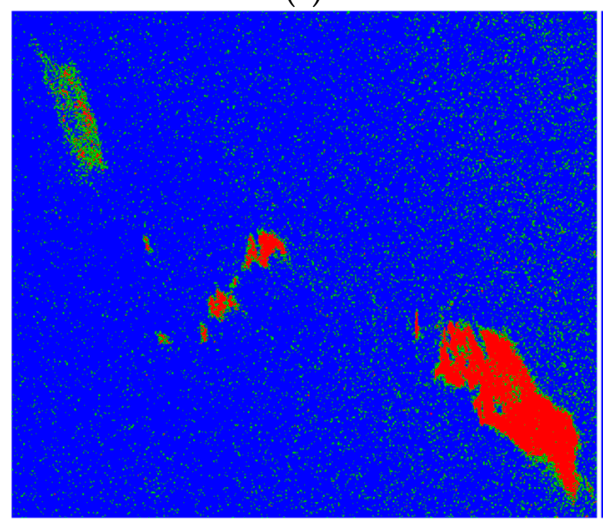

(d)

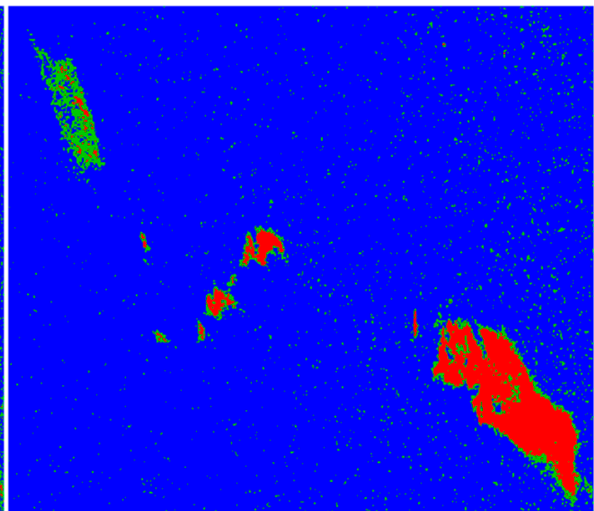

$(\mathrm{g})$

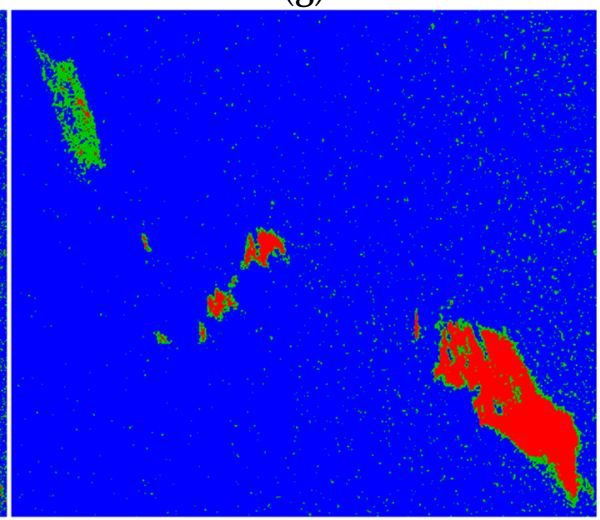

(h)

Figure 6. K-means Classifications of Radarsat-2 image into three classes. (a) Dop; (b) $\mu$; (c) $\log R c o$; (d) $\eta$; (e) $P h ;(\mathbf{f}) \log V ;$ (g) $A_{12}$; and (h) $r r r_{s}$.

In order to quantitatively compare these features, the Jeffreys-Matusita (J-M) distance was used to measure their oil spill detection capabilities. J-M distance is an index that is widely used to select features, which can evaluate well the ability of a feature to recognize a target [44]. In this paper, it was used to quantitatively analyze the oil spill detection ability of different polarimetric features. The expressions of J-M distance are shown as:

$$
\begin{gathered}
J=2\left(1-e^{-B}\right), \\
B=\frac{1}{8}\left(m_{1}-m_{2}\right)^{2} \frac{2}{\delta_{1}^{2}+\delta_{2}^{2}}+\frac{1}{2} \ln \left[\frac{\delta_{1}^{2}+\delta_{2}^{2}}{2 \delta_{1} \delta_{2}}\right],
\end{gathered}
$$

where $J$ represents the $\mathrm{J}-\mathrm{M}$ distance of features (such as $r r r_{s}, D o P$, etc.). $m_{1}$ and $m_{2}$ are the mean of the feature value of the two different targets, respectively. $\delta_{1}$ and $\delta_{2}$ are the standard deviation of the feature value of two different targets, respectively. The greater the value of the J-M distance, the stronger the distinguishability of the two targets. The J-M distances for the four different regions of eight polarimetric features on Radarsat-2 image are shown in Figure 7.

It can be seen in Figure 7 that the J-M distances of all polarimetric features are around 1.8 between the oil slicks and the water (c vs. $\mathrm{w}$ and e vs. w), proving that these eight features can both distinguish oil slicks from water very well. On the whole, among the four comparisons, the J-M distance of $r r r_{s}$ is the largest in all four comparisons. It can be concluded that the self-similarity parameter has better oil spill detection capability compared with other polarimetric features from the comparison of the three aspects above. 


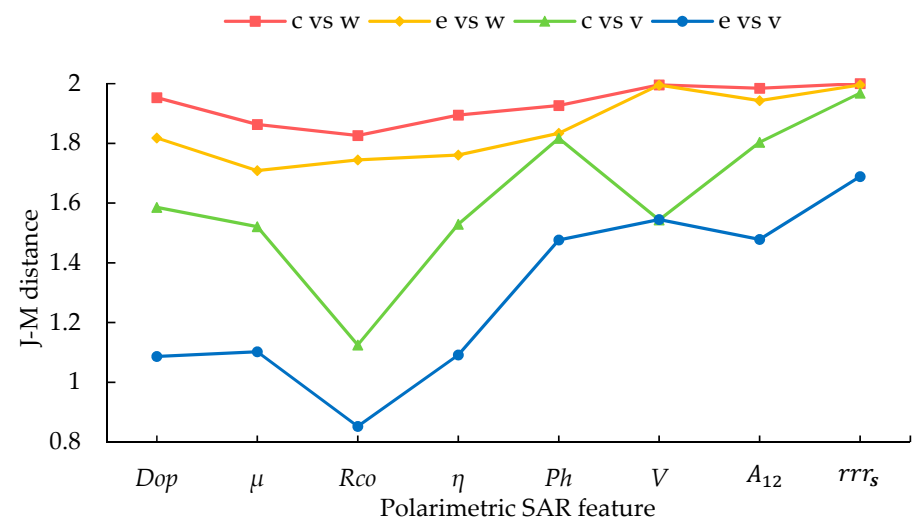

Figure 7. Jeffreys-Matusita (J-M) distances for eight polarimetric features in a Radarsat-2 image.

\subsection{Evaluation and Comparison}

In order to verify the improvement in oil spill detection accuracy by the proposed method, three well-known oil spill detection methods were compared with the proposed method in two polarimetric SAR datasets; namely: (1) the method which utilizes geometric intensity and the real part of the co-polarization cross product combined with $k$-means classification (GRK) [16]; (2) the method which utilizes the combined parameter F and Otsu segmentation (FOS) [14]; (3) the method which utilizes the extended Bragg model and Maximum likelihood classification (EBM) [26]; and (4) the proposed method which utilizes the self-similarity parameter and seven well-known features in combination with the RF classification (SRF).

In addition to qualitative visual evaluation, the oil spill detection results need quantitative evaluation. In this paper, the $F_{1}$ score was used to evaluate the quality of oil spill detection results. The $F_{1}$ score is an index used to measure the accuracy of the dichotomous model, which takes into account the accuracy and false alarm rate of the test results [45]. Expression of $F_{1}$ is calculated as:

$$
F_{1}=\frac{2 \times(1-F A R) \times D R}{(1-F A R+D R)},
$$

where $D R$ is the detection rate, and FAR is the false alarm rate.

According to the samples shown in Figures 3 and 4, the Radarsat- 2 image and UAVSAR image are classified by the proposed method, and classification results are shown in Figure 8. The Radarsat-2 image was classified into four classes: crude oil slicks (red), emulsion slicks (yellow), look-alikes (green), and water (blue), and the UAVSAR image was classified into three classes: emulsion slicks (yellow), look-alikes (green), and water (blue). The UAVSAR image selected here is the image labeled $\# 1$ in the second dataset.

As seen in Figure 8a,b, it is obvious that oil slicks, look-alikes, and water are successfully classified into three classes. In Figure 8a, both the crude oil areas and the emulsion areas are well classified as the oil slicks, and the vegetable oil areas in the upper left are classified as the look-alikes. In Figure 8b, both the three emulsion areas with different oil volumetric fractions are well classified as the oil slicks and the vegetable oil areas at the bottom are classified as the look-alikes. Hence, it can be concluded that the oil slicks and look-alikes have good classification results. However, there are still some misclassifications in the results. In Figure 8a, since the edge of crude oil and emulsion is subject to a large degree of weathering and diffusion, the outermost part of the crude oil and emulsion are classified as vegetable oil, which was also observed by Skrunes et al. [16]. The same phenomenon also appears in Figure 8b, a few pixels at the surrounding zones of emulsions are also classified as vegetable oil in the UAVSAR image, and a little vegetable oil is misclassified as emulsions.

According to the classification results, the look-alikes and water were masked and the crude oil slicks and emulsions were extracted as the final result of oil spill detection. Four methods were used to detect oil spills in two datasets. 


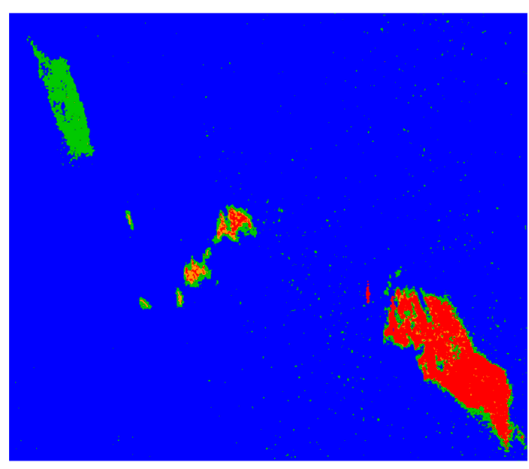

(a)

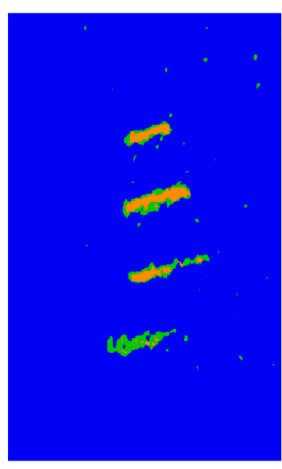

(b)

Figure 8. The Random Forest classification results. (a) Radarsat-2 image; and (b) UAVSAR image.

The oil spill detection results and accuracy measures of the Radarsat- 2 image using the different methods are shown in Figure 9 and Table 3, respectively. As shown in Figure 9, the crude oil and emulsion are detected well by the four methods. The main difference is the number of black spots on the upper left corner of the image, which is the false alarm caused by vegetable oil. Compared with the other three methods, SRF has the least number of black points in this region. As shown in Table 3, the $F_{1}$ score of SRF is over 3\% (FOS), $4 \%$ (GRK), and 12\% (EBM) higher than the other methods, respectively. It means that the proposed method obtains the best oil slicks' detection results in the Radarsat-2 image compared to the other three methods.

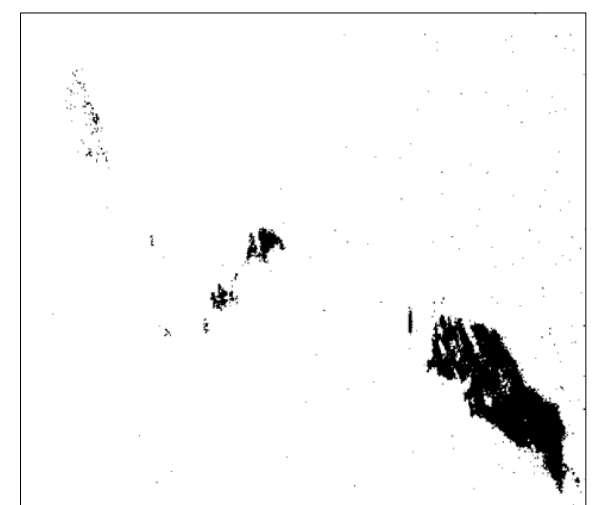

(a)

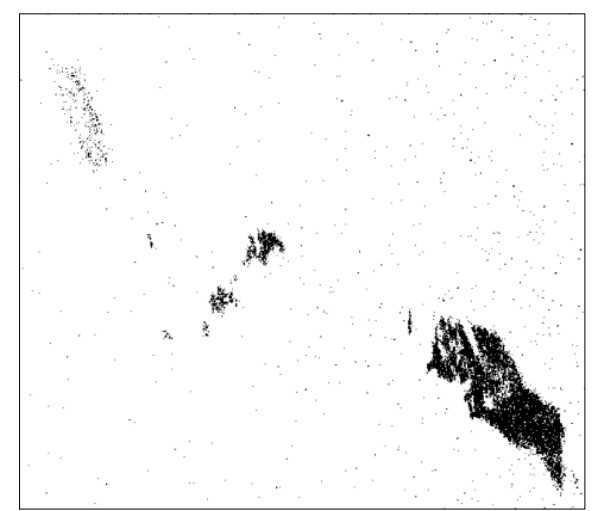

(c)

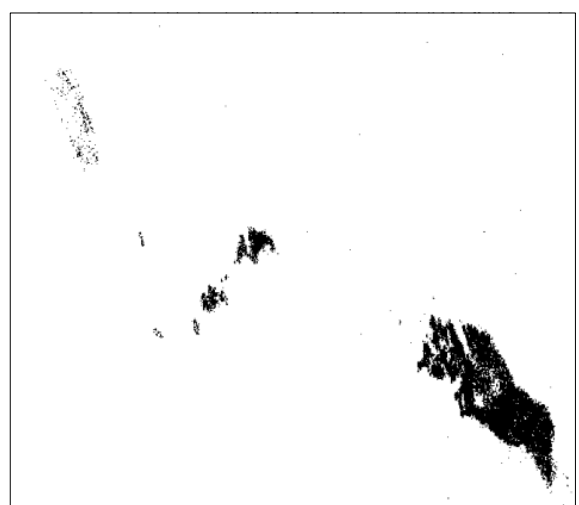

(b)

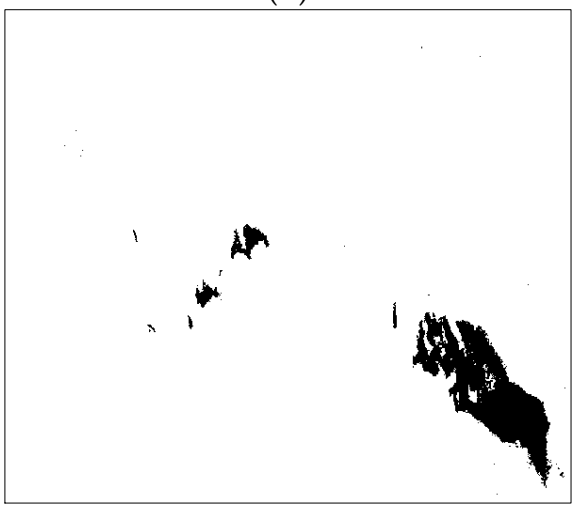

(d)

Figure 9. The oil spill detection results of four different methods in the Radarsat-2 polarimetric SAR image. (a) FOS; (b) GRK; (c) EBM; and (d) SRF. 
Table 3. The $F_{1}$ scores of four methods in the Radarsat-2 polarimetric SAR image.

\begin{tabular}{cccc}
\hline Method & DR & FAR & $\boldsymbol{F}_{1}$ \\
\hline FOS & $88.62 \%$ & $9.99 \%$ & $89.31 \%$ \\
GRK & $83.44 \%$ & $6.38 \%$ & $88.23 \%$ \\
EBM & $75.71 \%$ & $14.15 \%$ & $80.46 \%$ \\
SRF & $\mathbf{9 0 . 5 4 \%}$ & $\mathbf{4 . 4 3} \%$ & $\mathbf{9 2 . 9 9 \%}$ \\
\hline
\end{tabular}

The oil spill detection results and accuracy measures of the fourth scene UAVSAR image using the different methods are shown in Figure 10 and Table 4, respectively. As shown in Figure 10, three emulsions areas can be detected by the four methods, and the detection results in the GRK and the proposed method SRF are better, while the misclassifications in the FOS and EBM are more. In the look-alikes, the regions misclassified as oil slicks in the EBM and the proposed method SRF are less than in FOS and GRK. As shown in Table 4, it can be seen that the $F_{1}$ score of SRF is over 30\% (FOS), $4 \%$ (GRK) and 20\% (EBM) higher than other methods, respectively. It means that the proposed method obtains the best oil slicks detection result in the UAVSAR image compared to the other three methods.

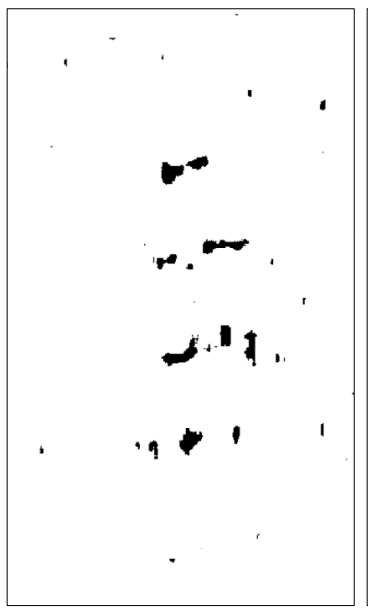

(a)

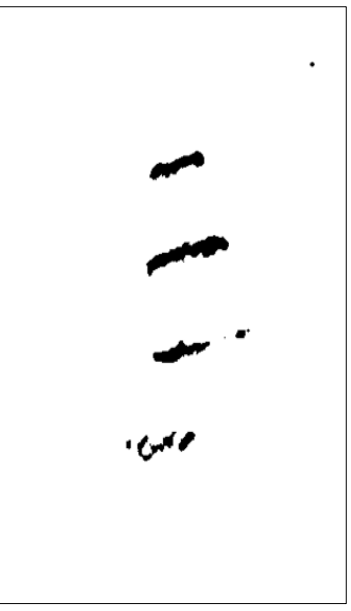

(b)

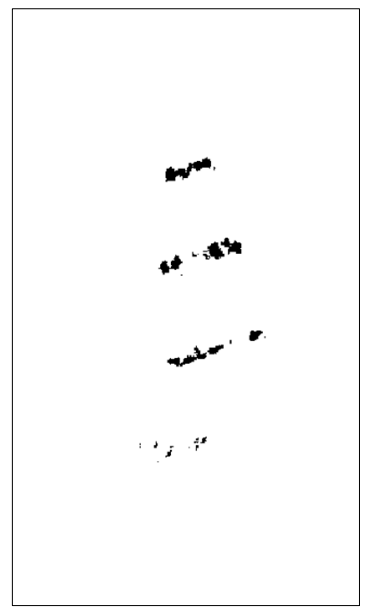

(c)

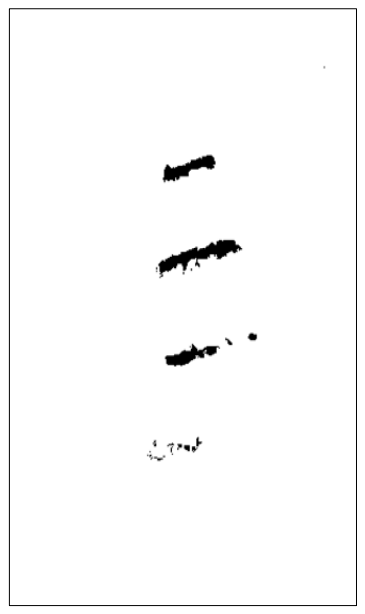

(d)

Figure 10. The oil spill detection results of four different methods in the UAVSAR polarimetric SAR image. (a) FOS; (b) GRK; (c) EBM; and (d) SRF.

Table 4. The $F_{1}$ scores of four methods in the UAVSAR polarimetric SAR image.

\begin{tabular}{cccc}
\hline Method & DR & FAR & $\boldsymbol{F}_{1}$ \\
\hline FOS & $45.63 \%$ & $40.70 \%$ & $51.57 \%$ \\
GRK & $\mathbf{7 6 . 9 6 \%}$ & $20.74 \%$ & $78.09 \%$ \\
EBM & $46.01 \%$ & $\mathbf{7 . 4 5 \%}$ & $61.46 \%$ \\
SRF & $74.47 \%$ & $8.15 \%$ & $\mathbf{8 2 . 2 5 \%}$ \\
\hline
\end{tabular}

In addition to the classification results, the RF also provides the VI for input features that can assess the importance of each feature in classification results. The VI values of eight polarimetric features are shown in Figure 11. Overall, the importance of the $\mathrm{rrr}_{s}$ is higher than that of other seven features, although it is closer to the $\mathrm{V}$ and $\mathrm{Ph}$ in Radarsat-2 image and closer to the $\mathrm{V}$ and Rco in UAVSAR image. It can be concluded that the self-similarity parameter has the highest contribution in RF classification for oil spill detection. In conclusion, it further demonstrates the advantages of the self-similarity parameter in oil spill detection. 


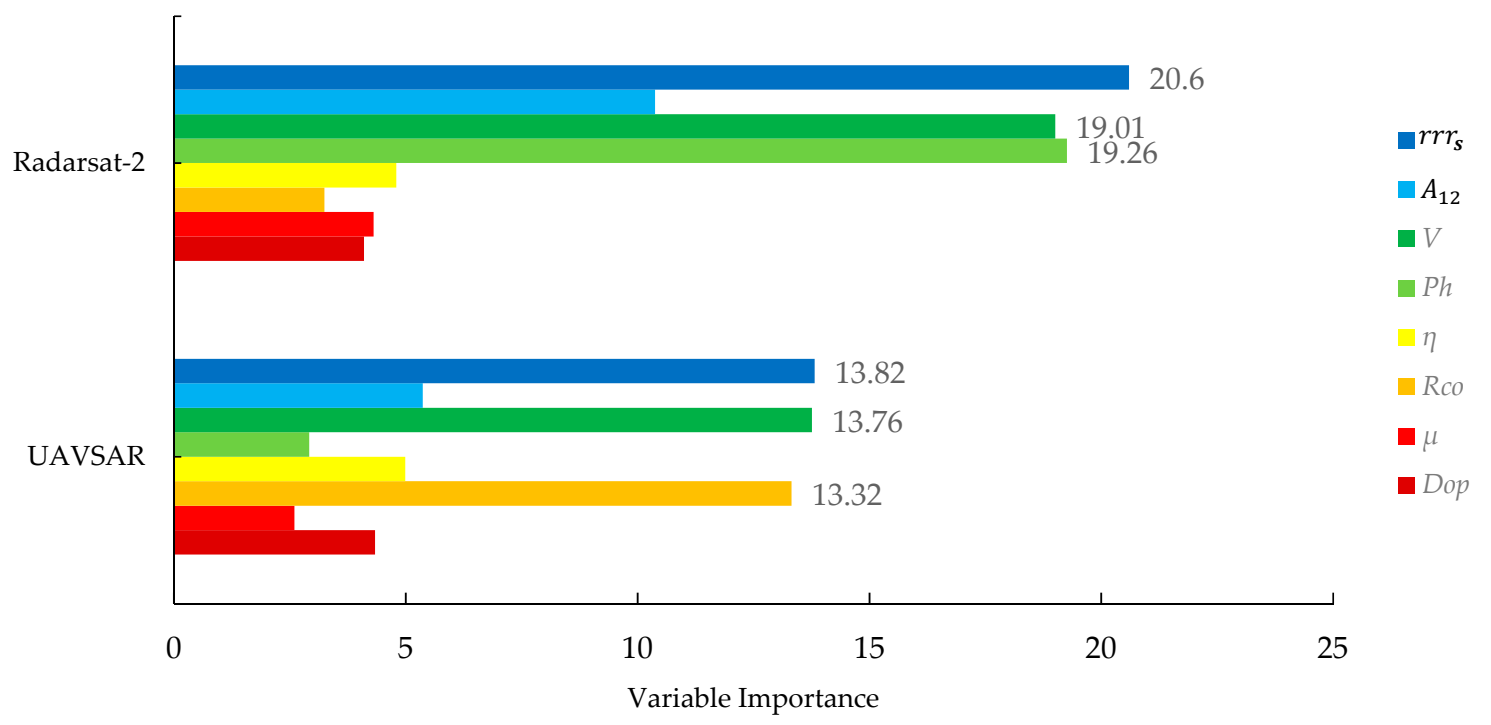

Figure 11. Contributions of eight features in the RF classification.

\section{Discussion}

\subsection{Noise Analysis}

In the case of polarimetric SAR oil spill detection, the returns from the oil slick and look-alike regions may be masked by sensor instrument noise for their backscatter signal may be lower than the sensor noise floor, i.e., Noise Equivalent Sigma Zero (NESZ). The NESZ should be lower than the returns to ensure that the information of each polarization channel is not interfered [46,47]. In such a situation, the self-similarity parameter and other polarimetric features which using full polarization information can accurately describe marine target and be used for oil spill detection. Hence, it is necessary to analyze the signal level over marine targets relative to the noise floor. Noise analyses are implemented based on the Radarsat-2 data and UAVSAR data, respectively.

As shown in Figure 12, the normalized radar cross section (NRCS) of each polarization channel $(\mathrm{HH}, \mathrm{VV}, \mathrm{VH})$ is plotted with the NESZ to analyze the signal level. The noise analyses of three polarization channels of Radarsat- 2 data with four groups of sample data, including crude oil, emulsion, vegetable oil, and water, are shown in Figure 12a-c. For the HH channel, the signal values of water are far greater than the NESZ. In the vegetable oil region, the signal values are lower than water but still lie above the NESZ. In the crude oil and emulsion regions, most of the signal values lie above the NESZ, but all of the signal values are lower than the vegetable oil. All the signal values of all sample data in the VV channel are higher than those in the HH channel, whereas, in the VH channel, most of the signal values of four regions lie below the NESZ, indicating that this channel is nearly affected by the instrument noise. The noise analyses of three polarization channels of UAVSAR data with five groups of sample data including E80, E60, E40, vegetable oil, and water, are shown in Figure 12d-f. As in the Radarsat-2 data, the ordering of the signal values in the UAVSAR data is water $>$ vegetable oil > oil slick, and VV channel $>\mathrm{HH}$ channel $>$ VH channel. The difference is that the signal values from both sample regions in the VH channel are higher than the NESZ since the UAVSAR has the lower NESZ. Based on the noise analyses presented here, the instrument noise has a small effect on the signals in the Radarsat-2 data, with the main impact being concentrated on the cross-polarization channel. In the UAVSAR data, each polarization channel signal is hardly affected by instrument noise. Hence, the self-similarity parameter and other polarimetric features used in this paper in these two data can describe the marine target well and detect oil spill. 


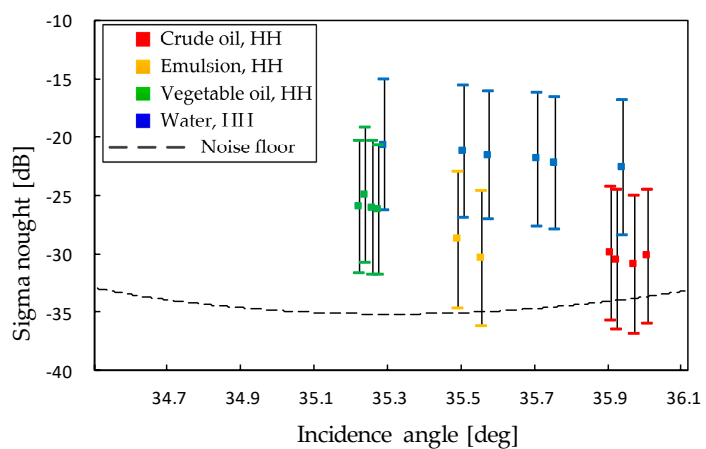

(a)

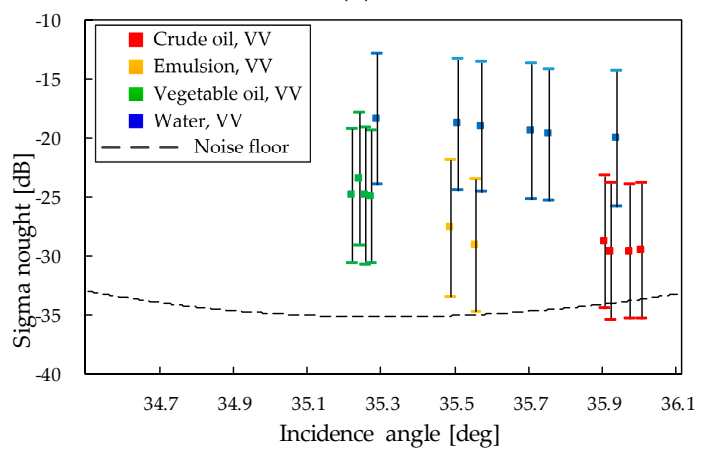

(b)

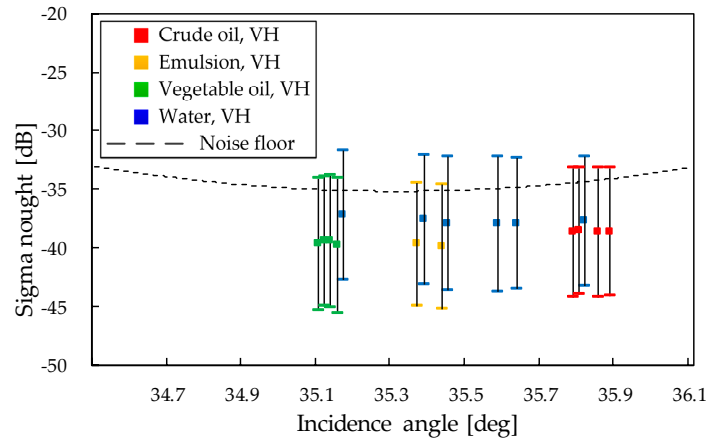

(c)

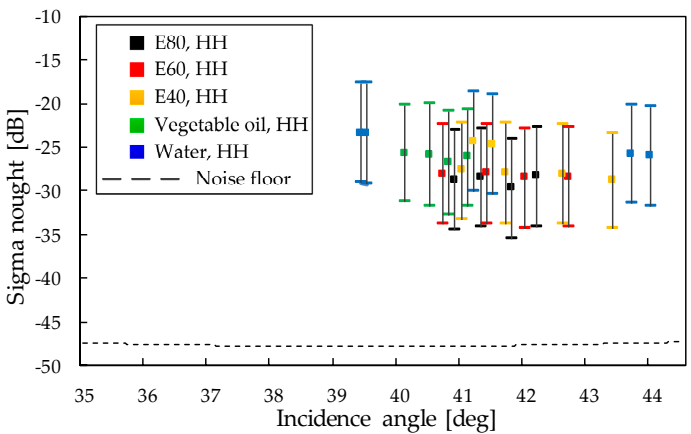

(d)

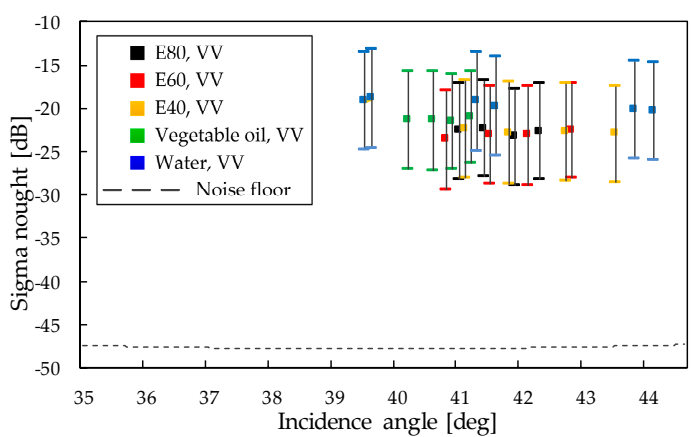

(e)

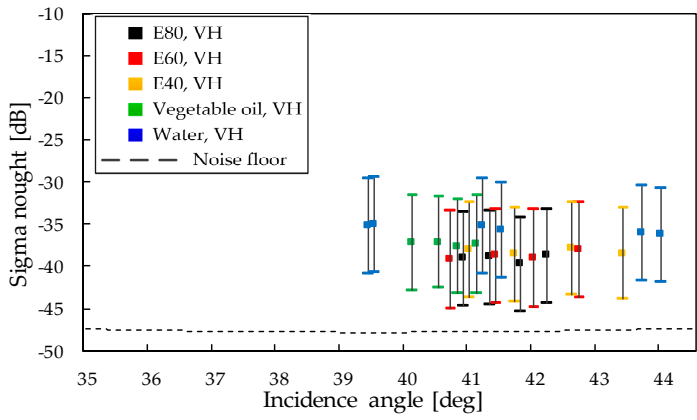

(f)

Figure 12. Signal-to-noise analyses of each channel of two sensors, the vertical bars show the mean and standard deviation of the backscatter values $\sigma_{0}$ in the regions indicated by samples in Figures 2 and 3. (a) HH channel (Radarsat-2); (b) VV channel (Radarsat-2); (c) VH channel (Radarsat-2); (d) HH channel (UAVSAR); (e) VV channel (UAVSAR); (f) VH channel (UAVSAR).

\subsection{Analysis of Oil Spill Detection Ability of Self-Similarity Parameter under Different Imaging Conditions}

In Section 3, we have analyzed the oil spill detection ability of each feature on the sea surface. It can be found that the polarimetric features can distinguish between the oil slicks and water, and the main difficulty of oil spill detection is to distinguish oil slicks from look-alikes. During the imaging process on the sea surface, the ability of polarimetric features to distinguish between oil slicks and look-alikes will be influenced by the different imaging conditions which have a certain impact on the scattering mechanism of the oil slicks [48]. The relevant factor analysis studies are in [48-50]. The main factors analyzed include the look direction relative to the wind, the sensor incidence angle, and the wind speed, etc. In this section, we mainly focus on the first two factors. The corresponding data is selected from the UAVSAR dataset to analyze the ability of the self-similarity parameter to distinguish between oil slicks and look-alikes under the different conditions of these two factors. 


\subsubsection{The Look Direction Relative to the Wind}

The look direction relative to the wind refers to the relative direction of the incident direction of the SAR and the wind direction of the sea surface and is divided into two scenes: downwind and upwind. The nonpolarized component has been shown to account for most of the differences observed between upwind and downwind backscatter [48]. Hence, the ability of polarimetric feature distinguishes between oil slicks and look-alikes may be different in different wind directions. In this paper, two scenes of upwind and downwind images with similar imaging time and incidence angle in the oil slick areas were used for the comparative experiment, i.e., \#2 and \#3. The J-M distances between the oil slicks and the look-alikes of the polarimetric features in these two scenes are shown in Figure 13.

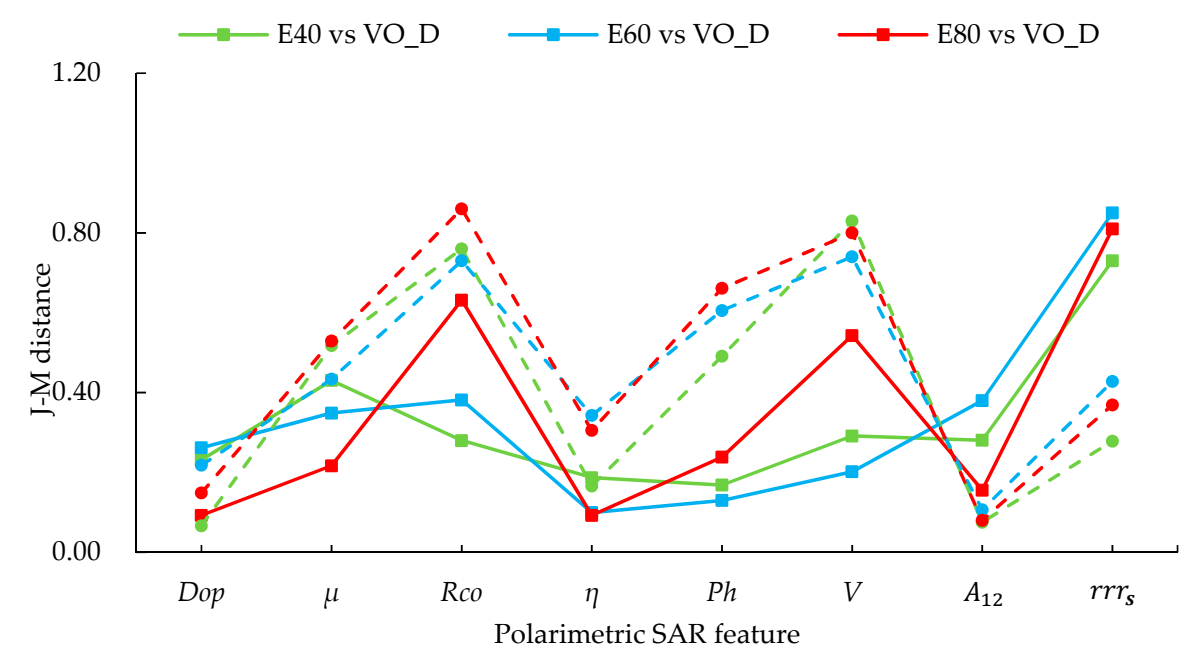

Figure 13. The J-M distances between the oil slicks and the look-alikes of the polarimetric features in different wind direction scenes. E40, E60, and E80 represent the oil slicks with oil volumetric fractions of $40 \%, 60 \%$, and $80 \%$, respectively, VO represents the vegetable oil, the suffix D represents the downwind and $U$ represents the upwind.

It can be seen from Figure 13 that the ability of the self-similarity parameter to distinguish between oil slicks and look-alikes varies widely in different wind directions. The discrimination ability of the self-similarity parameter in the downwind is significantly stronger than the upwind. In the downwind scene, the ability of the self-similarity parameter to distinguish between oil slicks and look-alikes is stronger than other polarimetric features, while, in the upwind scene, it is weaker than the Rco, V, etc. It means that the self-similarity parameter is more suitable for distinguishing oil slicks and look-alikes in the downwind scene than other polarimetric features, while it cannot offer better distinguishability than some features in the upwind scene.

\subsubsection{The Sensor Incidence Angle}

The relevant studies proved that the values of the polarimetric feature in each region (e.g., oil slick, water, look-alike, etc.) are different at different incidence angle conditions [49,50]. Hence, the ability of polarimetric feature distinguish between oil slicks and look-alikes may differ in different incidence angles. Two images with similar imaging time and the same wind direction were used for the comparative experiment, i.e., \#1 and \#2, to analyze the ability to distinguish between oil slicks and look-alikes of self-similarity parameter under different incidence angle conditions. The J-M distances between the oil slicks and the look-alikes of the polarimetric features in these two scenes are shown in Figure 14.

It can be seen from Figure 14 that the ability of the self-similarity parameter to distinguish oil slicks from the look-alikes has little difference in these two angles of incidence scenes. The discrimination ability of the self-similarity parameter in the scene with the incidence angle ranges from $29.7^{\circ}$ to $34.4^{\circ}$ is 
slightly stronger than that from $39.6^{\circ}$ to $43.5^{\circ}$. In addition, in the $29.7^{\circ}$ to $34.4^{\circ}$ scene, the discrimination ability of the self-similarity parameter is significantly higher than other polarimetric features. In the $39.6^{\circ}$ to $43.5^{\circ}$ scene, the discrimination ability of the self-similarity parameter is close to that of Rco and V, and is higher than that of other features. It means that the self-similarity parameter maintains a good ability to distinguish between oil slicks and look-alikes in both of these two angles of incidence scenes.

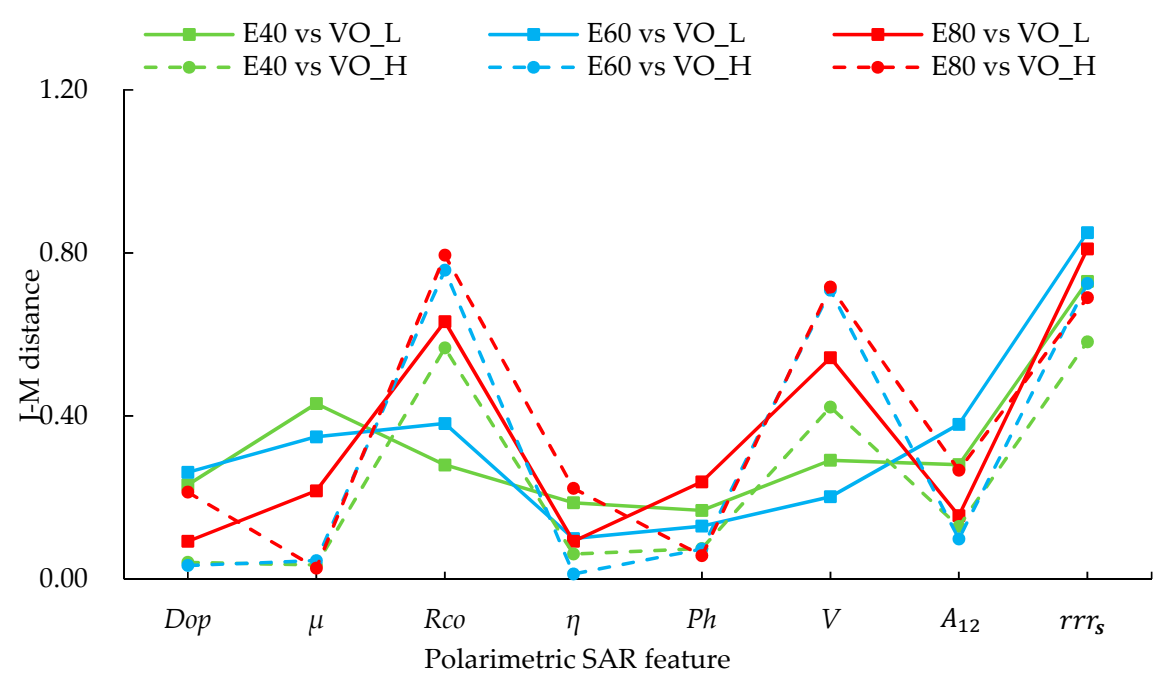

Figure 14. The J-M distances between the oil slicks and the look-alikes of the polarimetric features in different incidence angle scenes. The suffix $\mathrm{H}$ indicates higher incidence angle scene (the incidence angle of the oil slick areas ranges from $39.6^{\circ}$ to $43.5^{\circ}$ ), and $\mathrm{L}$ indicates lower incidence angle scene (the incidence angle of the oil slick areas ranges from $29.7^{\circ}$ to $34.4^{\circ}$ ).

\subsection{Accuracies, Errors, and Uncertainties}

The above experiment results prove that the proposed method has a better effect of oil spill detection compared to the other three methods. Nevertheless, there are some factors that may affect the oil spill detection result of the proposed method, such as the degree of weathering of the oil slicks, sea surface wind speed, incidence angles of oil slick areas, etc.

The oil slicks covering the sea surface are affected by weathering, which includes the evaporation, emulsification, diffusion, dissolution, oxidation, etc. They will change the physical and chemical properties of the oil slicks, which influence the scattering mechanism. From the classification results of Radarsat-2 images, it can be seen that the parts of emulsions with longer weathering time that is misclassified as the look-alikes are more than the fresh crude oil slicks. From the classification results of UAVSAR images, it can be seen that the parts of the emulsions with different oil volume fractions that are misclassified as look-alikes are also different. Therefore, the degree of emulsification of the oil slicks will bring certain error effects on the experimental results.

Two kinds of polarimetric SAR data with different wind speeds were used to evaluate the effect of the oil spill detection of the proposed method. Nevertheless, the specific impact of different wind speeds on the result of oil spill detection is unclear. It can be seen from the experiment results that the J-M distances between the oil slicks and look-alikes of polarimetric features in UAVSAR images with high wind speed are generally lower than that of the Radarsat- 2 image, and the former accuracy of the oil spill detection is also lower than the latter. Wind speed is one of the important factors that cause this difference. Nonetheless, it is difficult to judge the contribution of wind speed in this difference, since the two images have different sensor parameters in addition to the wind speed (e.g., sensor band and instrument noise floor, etc.). Therefore, the impact of wind speed on the oil detection capability of the proposed method and related polarimetric features requires further analysis based on relevant data. 
In addition, the incidence angles of oil slick areas in the experimental data range from $29.7^{\circ}$ to $43.5^{\circ}$. Nevertheless, the UAVSAR can provide a larger incidence angle, i.e., $19.5-67.5^{\circ}$. Hence, the oil spill detection capability of the proposed method and the self-similarity parameter outside $29.7^{\circ}$ to $43.5^{\circ}$ requires more relevant data for analysis.

\section{Conclusions}

Oil spill identification in single-polarization SAR imagery is a challenging task due to the presence of look-alikes, which have surface signatures similar to oil spills. Nonetheless, this task is made easier by exploiting polarimetric SAR data using proper modelling tools. In this paper, a novel multi-feature based ocean oil spill detection method for polarimetric SAR data has been proposed by using Random Forest (RF) classification. This method combines the self-similarity parameter and seven other polarimetric features. The main characteristics are as follows: first, RF classification utilizes a variety of polarimetric features of oil spill detection to distinguish oil slicks from look-alikes accurately. Second, introducing a self-similarity parameter with better oil detection capabilities further improves the detection accuracy.

Two kinds of different scenarios of oil spill datasets (Radarsat-2 and UAVSAR) were utilized to validate the practicability of the proposed method. Experiments show that the accuracy of the proposed method reaches $92.99 \%$ and $82.25 \%$, respectively, which is higher than the three well-known methods in these datasets. It can be concluded that the proposed method improves the accuracy of oil spills effectively. In addition, the oil spill detection ability of polarimetric features was measured and compared. The results prove that the self-similarity parameter has the better oil spill detection capability compared other seven polarimetric features in the scene with lower wind speed, and performs well in the scene with incidence angle range from $29.7^{\circ}$ to $43.5^{\circ}$.

Nevertheless, for extreme sea conditions or different degrees of weathering slicks, the performance of the proposed method may be affected. Hence, the impact of wind speed on the oil detection capability, the quantitative analysis of the weathering effect of oil slicks on oil spill detection accuracy and the detection ability of the scenes outside the incidence angle, which ranges from $29.7^{\circ}$ to $43.5^{\circ}$, requires further analysis with more relevant data.

Author Contributions: S.T. drafted the manuscript and was responsible for the research design and experiment; X.L., Z.Z., and G.X. reviewed the manuscript and were responsible for the analysis; Q.C. supervised the research and contributed to the editing and review of the manuscript.

Funding: This work is supported in part by the National Natural Science Foundation of China under Grant No. 41771467, No. 41471355, and the Fundamental Research Funds for the Central Universities, China University of Geosciences (Wuhan) No. CUG160707.

Acknowledgments: The authors would like to thank the NASA for providing the UAVSAR quad-pol SAR images. We also thank Linlin Li, Xiaoli Xing and Shuai Yang for their support during the entire course of this study.

Conflicts of Interest: The authors declare no conflict of interest.

\section{References}

1. Fingas, M.; Brown, C.E. Handbook of Oil Spill Science and Technology; John Wiley and Sons: Hoboken, NJ, USA, 2015.

2. Brekke, C.; Solberg, A.H.S. Oil spill detection by satellite remote sensing. Remote Sens. Environ. 2005, 95, 1-13. [CrossRef]

3. Nunziata, F.; Migliaccio, M.; Gambardella, A. Pedestal height for sea oil slick observation. IET Radar. Sonar. Nav. 2011, 5, 103. [CrossRef]

4. Migliaccio, M.; Nunziata, F.; Gambardella, A. On the Copolarised Phase Difference for Oil Spill Observation. Int. J. Remote Sens. 2007, 6, 1587-1602.

5. Kubat, M.; Holte, R.C.; Matwin, S. Machine Learning for the Detection of Oil Spills in Satellite Radar Images. Mach. Learn. 1998, 30, 195-215. [CrossRef] 
6. Migliaccio, M.; Tranfaglia, M.; Ermakov, S.A. A physical approach for the observation of oil spills in SAR images. IEEE J. Oceanic Eng. 2005, 30, 496-507. [CrossRef]

7. Migliaccio, M.; Gambardella, A.; Tranfaglia, M. SAR Polarimetry to Observe Oil Spills. IEEE Trans. Geosci. Remote Sens. 2007, 45, 506-511. [CrossRef]

8. Latini, D.; Del Frate, F.; Jones, C.E. Multi-frequency and polarimetric quantitative analysis of the Gulf of Mexico oil spill event comparing different SAR systems. Remote Sens. Environ. 2016, 183, 26-42. [CrossRef]

9. Liu, P.; Zhao, C.; Li, X.; He, M.; Pichel, W. Identification of ocean oil spills in SAR imagery based on fuzzy logic algorithm. Int. J. Remote Sens. 2010, 31, 4819-4833. [CrossRef]

10. Buono, A.; Nunziata, F.; Migliaccio, M.; Li, X. Polarimetric Analysis of Compact-Polarimetry SAR Architectures for Sea Oil Slick Observation. IEEE Trans. Geosci. Remote Sens. 2016, 54, 5862-5874. [CrossRef]

11. Song, D.; Ding, Y.; Li, X.; Zhang, B.; Xu, M. Ocean Oil Spill Classification with RADARSAT-2 SAR Based on an Optimized Wavelet Neural Network. Remote Sens. 2017, 9, 799. [CrossRef]

12. Salberg, A.; Larsen, S.O. Classification of Ocean Surface Slicks in Simulated Hybrid-Polarimetric SAR Data. IEEE Trans. Geosci. Remote Sens. 2018, 56, 7062-7073. [CrossRef]

13. Migliaccio, M.; Nunziata, F.; Buono, A. SAR polarimetry for sea oil slick observation. Int. J. Remote Sens. 2015, 36, 3243-3273. [CrossRef]

14. Liu, P.; Li, X.; Qu, J.J.; Wang, W.; Zhao, C.; Pichel, W. Oil spill detection with fully polarimetric UAVSAR data. Mar. Pollut Bull. 2011, 62, 2611-2618. [CrossRef] [PubMed]

15. Ramsey, E., III; Rangoonwala, A.; Suzuoki, Y.; Jones, C.E. Oil Detection in a Coastal Marsh with Polarimetric Synthetic Aperture Radar (SAR). Remote Sens. 2011, 3, 2630-2662. [CrossRef]

16. Skrunes, S.; Brekke, C.; Eltoft, T. Characterization of Marine Surface Slicks by Radarsat-2 Multipolarization Features. IEEE Trans. Geosci. Remote Sens. 2014, 52, 5302-5319. [CrossRef]

17. Fan, Y.; Jian, Y.; Yin, J.; Jian, S. Spill detection based on polarimetric SAR decomposition models. J. Tsinghua Univ. 2015, 854-859.

18. Zhang, B.; Perrie, W.; Li, X.; Pichel, W.G. Mapping sea surface oil slicks using RADARSAT-2 quad-polarization SAR image. Geophys Res. Lett. 2011, 38, 415-421. [CrossRef]

19. Skrunes, S.; Brekke, C.; Eltoft, T.; Kudryavtsev, V. Comparing Near-Coincident C- and X-Band SAR Acquisitions of Marine Oil Spills. IEEE Trans. Geosci. Remote Sens. 2014, 53, 1958-1975. [CrossRef]

20. Nunziata, F.; Gambardella, A.; Migliaccio, M. On the degree of polarization for SAR sea oil slick observation. ISPRS J. Photogramm. Remote Sens. 2013, 78, 41-49. [CrossRef]

21. Kumar, L.J.V.; Kishore, J.K.; Rao, P.K. Decomposition methods for detection of oil spills based on Risat-1 SAR images. Int. J. Remote Sens. Geosci. 2014, 3, 1-10.

22. Alpers, W.; Holt, B.; Zeng, K. Oil spill detection by imaging radars: Challenges and pitfalls. Remote Sens. Environ. 2017, 201, 133-147. [CrossRef]

23. Zhang, B.; Li, X.; Perrie, W.; Garcia-Pineda, O. Compact Polarimetric Synthetic Aperture Radar for Marine Oil Platform and Slick Detection. IEEE Trans. Geosci. Remote Sens. 2017, 55, 1-17. [CrossRef]

24. Li, Y.; Lin, H.; Zhang, Y.; Chen, J. Comparisons of Circular Transmit and Linear Receive Compact Polarimetric SAR Features for Oil Slicks Discrimination. J. Sens. 2015, 2015, 1-14. [CrossRef]

25. Shirvany, R.; Chabert, M.; Tourneret, J.Y. Ship and Oil-Spill Detection Using the Degree of Polarization in Linear and Hybrid/Compact Dual-Pol SAR. IEEE J.-Stars. 2012, 5, 885-892. [CrossRef]

26. Yin, J.; Yang, J.; Zhou, Z.; Song, J. The Extended Bragg Scattering Model-Based Method for Ship and Oil-Spill Observation Using Compact Polarimetric SAR. IEEE J.-Stars. 2015, 8, 3760-3772. [CrossRef]

27. Guo, H.; Wu, D.; An, J. Discrimination of Oil Slicks and Lookalikes in Polarimetric SAR Images Using CNN. Sensors 2017, 17, 1837. [CrossRef] [PubMed]

28. Mahdianpari, M.; Salehi, B.; Mohammadimanesh, F.; Motagh, M. Random forest wetland classification using ALOS-2 L-band, RADARSAT-2 C-band, and TerraSAR-X imagery. ISPRS J. Photogramm. Remote Sens. 2017, 130, 13-31. [CrossRef]

29. Wang, W.; Yang, X.; Li, X.; Chen, K.; Liu, G.; Li, Z.; Gade, M. A Fully Polarimetric SAR Imagery Classification Scheme for Mud and Sand Flats in Intertidal Zones. IEEE Trans. Geosci. Remote Sens. 2017, 55, 1734-1742. [CrossRef]

30. Chen, W.; Li, X.; He, H.; Wang, L. Assessing Different Feature Sets' Effects on Land Cover Classification in Complex Surface-Mined Landscapes by ZiYuan-3 Satellite Imagery. Remote Sens. 2017, 10, 23. [CrossRef] 
31. Chen, W.; Li, X.; He, H.; Wang, L. A Review of Fine-Scale Land Use and Land Cover Classification in Open-Pit Mining Areas by Remote Sensing Techniques. Remote Sens. 2017, 10, 15. [CrossRef]

32. Li, D.; Zhang, Y. Random Similarity Between Two Mixed Scatterers. IEEE Geosci. Remote Sens Lett. 2015, 12, 2468-2472.

33. Tong, S.; Chen, Q.; Liu, X. Sea oil spill detection using self-similarity parameter of polarimetric sar data. Int. Arch. Photogramm. Remote Sens. Spat. Inform. Sci. 2018, 42, 1661-1666.

34. Breiman, L. Random Forests. Mach. Learn. 2001, 45, 5-32. [CrossRef]

35. Yang, J.; Peng, Y.N.; Lin, S.M. Similarity between two scattering matrices. Electron. Lett. 2001, 37, $193-194$. [CrossRef]

36. Chen, Q.; Jiang, Y.M.; Zhao, L.J.; Kuang, G.Y. Polarimetric Scattering Similarity Between a Random Scatterer and a Canonical Scatterer. IEEE Geosci. Remote Sens Lett. 2010, 7, 866-869. [CrossRef]

37. Nunziata, F.; Sobieski, P.; Migliaccio, M. The Two-Scale BPM Scattering Model for Sea Biogenic Slicks Contrast. IEEE Trans. Geosci. Remote Sens. 2009, 47, 1946-1956. [CrossRef]

38. Nunziata, F.; Gambardella, A.; Migliaccio, M. On the Mueller Scattering Matrix for SAR Sea Oil Slick Observation. IEEE Geosci. Remote Sens Lett. 2008, 5, 691-695. [CrossRef]

39. Zou, T.; Yang, W.; Dai, D.; Sun, H. Polarimetric SAR Image Classification Using Multifeatured Combination and Extremely Randomized Clustering Forests. Eurasip J. Adv. Signal Pr. 2009, 2010, 1-9.

40. Zhao, L.; Yang, J.; Li, P.; Huang, X.; Shi, L.; Zhang, L. Characterization of the coherent scattering induced by ridging patterns in agriculture by the use of polarimetric SAR imagery. Int. J. Remote Sens. 2017, 38, 3502. [CrossRef]

41. Houborg, R.; Mccabe, M.F. A hybrid training approach for leaf area index estimation via Cubist and random forests machine-learning. ISPRS J. Photogramm. Remote Sens. 2017, 135, 173-188. [CrossRef]

42. Rossel, R.A.V.; Behrens, T.; Guerrero, C.; Viscarra Rossel, R.A.; Mouazen, A.M. Using data mining to model and interpret soil diffuse reflectance spectra. Geoderma 2010, 158, 46-54. [CrossRef]

43. Espeseth, M.M.; Skrunes, S.; Jones, C.E.; Brekke, C.; Holt, B.; Doulgeris, A.P. Analysis of Evolving Oil Spills in Full-Polarimetric and Hybrid-Polarity SAR. IEEE Trans. Geosci. Remote Sens. 2017, 55, 1-21. [CrossRef]

44. Dabboor, M.; Howell, S.; Shokr, M.; Yackel, J. The Jeffries-Matusita distance for the case of complex Wishart distribution as a separability criterion for fully polarimetric SAR data. Int J. Remote Sens. 2014, 35, 6859-6873.

45. Powers, D.M.W. Evaluation: From Precision, Recall and F-Factor to ROC, Informedness, Markedness \& Correlation. J. Mach. Learn. Technol. 2011, 2, 2229-3981.

46. Li, G.; Li, Y.; Liu, B.; Hou, Y.; Fan, J. Analysis of Scattering Properties of Continuous Slow-Release Slicks on the Sea Surface Based on Polarimetric Synthetic Aperture Radar. Isprs Int. J. Geo-Inf. 2018, 7, 237. [CrossRef]

47. Skrunes, S.; Brekke, C.; Jones, C.E.; Holt, B. A Multi-sensor Comparison of Experimental Oil Spills in Polarimetric SAR for High Wind Conditions. IEEE J.-Stars. 2016, 9, 4948-4961.

48. Skrunes, S.; Brekke, C.; Jones, C.E.; Espeseth, M.M.; Holt, B. Effect of wind direction and incidence angle on polarimetric SAR observations of slicked and unslicked sea surfaces. Remote Sens. Environ. 2018, 213, 73-91. [CrossRef]

49. Minchew, B.; Jones, C.E.; Holt, B. Polarimetric Analysis of Backscatter from the Deepwater Horizon Oil Spill Using L-Band Synthetic Aperture Radar. IEEE Trans. Geosci. Remote Sens. 2012, 50, 3812-3830. [CrossRef]

50. Buono, A.; Nunziata, F.; de Macedo, C.R.; Velotto, D.; Migliaccio, M. A Sensitivity Analysis of the Standard Deviation of the Copolarised Phase Difference for Sea Oil Slick Observation. IEEE Trans. Geosci. Remote Sens. 2018, 1-9. [CrossRef]

(C) 2019 by the authors. Licensee MDPI, Basel, Switzerland. This article is an open access article distributed under the terms and conditions of the Creative Commons Attribution (CC BY) license (http://creativecommons.org/licenses/by/4.0/). 